\title{
Walk to me when I smile, step back when I'm angry: emotional faces modulate whole-body approach-avoidance behaviors
}

\author{
John F. Stins $\cdot$ Karin Roelofs $\cdot$ Jody Villan • \\ Karen Kooijman • Muriel A. Hagenaars • \\ Peter J. Beek
}

Received: 7 January 2011 / Accepted: 9 June 2011/Published online: 23 June 2011

(C) The Author(s) 2011. This article is published with open access at Springerlink.com

\begin{abstract}
Facial expressions are potent social cues that can induce behavioral dispositions, such as approachavoidance tendencies. We studied these tendencies by asking participants to make whole-body forward (approach) or backward (avoidance) steps on a force plate in response to the valence of social cues (happy or angry faces) under affect-congruent and incongruent mappings. Posturographic parameters of the steps related to automatic stimulus evaluation, step initiation (reaction time), and step execution were determined and analyzed as a function of stimulus valence and stimulus-response mapping. The main result was that participants needed more time to initiate a forward step towards an angry face than towards a smiling face (which is evidence of a congruency effect), but with backward steps, this difference failed to reach significance. We also found a reduction in spontaneous body sway prior to the step with the incongruent mapping. The results provide a crucial empirical link between theories of socially induced action tendencies and theories of postural control and suggest a motoric basis for socially guided motivated behavior.
\end{abstract}

Keywords Emotional facial expression . Postural control - Emotion - Reaction time . Step initiation $\cdot$ Approach-avoidance

J. F. Stins $(\bowtie) \cdot$ J. Villan · K. Kooijman · P. J. Beek Research Institute MOVE, Faculty of Human Movement Sciences, VU University Amsterdam, van der Boechorststraat 9, 1081 BT Amsterdam, The Netherlands e-mail: j.stins@fbw.vu.nl

K. Roelofs · M. A. Hagenaars

Behavioural Science Institute (BSI), Radboud University

Nijmegen, Nijmegen, The Netherlands
Emotions are considered action dispositions that were shaped in the course of evolution (e.g., Bradley et al. 2001a; Lang et al. 1998). A core distinction is that between aversive and appetitive motivations, which trigger approach and avoidance behavior in response to salient environmental events. Approach behaviors are elicited by pleasant or desirable situations, for example, the presence of food, a potential sexual mate, or a novel object that invites exploration. Avoidance behaviors can be elicited by dangerous or threatening situations, such as a predator or the sight of a dead conspecific. Most theoretical positions assume that valence of stimuli is processed automatically, and that valence induces behavioral dispositions. These dispositions can be revealed using manual approach-avoidance (AA) tasks where participants respond to visually presented appetitive and aversive cues by moving their arm either towards (approach) or away from (avoid) their body. These choice reaction time tasks (e.g., Chen and Bargh 1999; Eder and Rothermund 2008; Rinck and Becker 2007; Roelofs et al. 2005) contrast two response conditions. In the affect-congruent response condition, participants are instructed to approach appetitive and to avoid aversive stimuli, and in the affect-incongruent condition they have to approach aversive and to avoid appetitive cues. The majority of studies have found faster and more accurate reactions in the affect-congruent condition than the incongruent one, where participants have to override their "instinctive" or "intuitive" action tendencies (Roelofs et al. 2009a). In addition, approach-avoidance tendencies are further modulated by subject variables such as anxiety (Rinck and Becker 2007), and motivational variables related to maximization of gain and/or minimization of loss, i.e., "regulatory focus" (e.g., Förster et al. 1998, 2001). 
Approach-avoidance tasks typically employ unimanual one-degree-of-freedom movements involving arm flexions (signifying approach) and arm extensions (signifying avoidance). Arm flexions and extensions can involve pressing one of two buttons aligned in a row (e.g., Roelofs et al. 2005), pulling or pushing a joystick (e.g., Chen and Bargh 1999), or pushing upward against the bottom of a table (using the arm flexors) versus pushing downward against a table, using the arm extensors (e.g., Förster et al. 2001). But all these paradigms carry the potential disadvantage that congruency effects are critically dependent on subjects' cognitive interpretation of the task, which possibly reduces the ecological validity of the arm flexionextension setup; for example, Markman and Brendl (2005) found that subjects were faster in moving a positive word toward their name on a screen and moving a negative word away from their name on a screen than with the converse assignment. Importantly, this effect was independent of the actual movement direction (push vs. pull) of the arm. This finding was taken to suggest that congruency effects depend on the representation of the task, rather than the physical characteristics of the movement. Relatedly, Van Peer et al. (2010) argued that the link between affectevaluation and the execution of a response is not direct, but is likely mediated by symbolic representations. With respect to the use of joysticks in AA tasks, it has been argued that joystick movements are ambiguous regarding their distance-changing consequences (e.g., Krieglmeyer et al. 2010). These latter authors pointed out that pushing a joystick can either signify pushing away from the body (i.e., avoidance) or reaching out the hand to grab something (i.e., approach). This ambiguity likely increases error variance.

Over the past few years, several solutions circumventing these ambiguities have been developed. Some authors give explicit instructions to move the joystick "towards their body" or "away from their body", while others opt for more implicit manipulations of approach and avoidance (Roelofs et al. 2009a). Krieglmeyer et al. (2010) instructed participants to move a manikin in a particular direction (see also Lavender and Hommel 2007) and Rinck and Becker (2007) developed a so-called "zooming" version of the approach-avoidance task whereby the visual stimuli lawfully changed in apparent size upon deflection of the joystick. This generates a visual impression of a picture coming towards or moving away from the participant, preventing ambiguity in the distance-changing consequences and making them resistant to cognitive reinterpretations (Rinck and Becker 2007). Still, the unimanual arm flexion/extension approaches remain indirect, representing altered distance via representation of the self or the other as displayed on a monitor. In this study, we propose a more direct measure of approach-avoidance behavior, based on whole-body movements toward or away from the stimulus.

Koch et al. (2009) stated that "In the most fundamental and literal sense, approach refers to decreasing and avoidance to increasing the physical distance between the self and the outside world." (p. 549). However, and somewhat surprisingly, no study to date using an AA task has operationalized "approach" and "avoidance" in terms of an actual change in physical proximity between the body and the eliciting event. The aim of this study was to investigate whether approach-avoidance tendencies emerge using whole-body stepping movements toward or away from facial expressions signifying anger or happiness. Self-generated forward or backward body locomotion may provide a unique opportunity to study approach-avoidance behavior to social cues in its "purest" form (Koch et al. 2009). In addition, forward or backward stepping automatically generates the expected sensory consequences of increasing or decreasing the visual angle of the stimulus, as the change in visual angle is a natural consequence of the stepping movements. Thus, cued forward/backward stepping involves a novel setup with high ecological validity, both in terms of the sensory consequences of the movements and in terms of actual whole-body approach/avoidance movements that arguably leave no room for cognitive reinterpretation.

We decided to study approach-avoidance behavior in response to facial expressions. Several studies (e.g., Marsh et al. 2005; Roelofs et al. 2009a; Rotteveel and Phaf 2004; Seidel et al. 2010) have found reliable congruency effects in response to the emotion category of the facial expression. As pointed out by Seidel et al. (2010), faces are powerful social cues that signal the emotional state and behavioral intentions of the poser. The behavioral data of Seidel et al. (2010) revealed approach tendencies with happy faces and avoidance tendencies (although non-significant) with angry faces. Interestingly, the latter study already hinted at the relevance of studying forward/backward whole-body movements on account of the subjective rating procedure adopted. Seidel et al. (2010) asked subjects to rate their approach-avoidance tendencies of emotional expressions by scoring how many steps toward or away from a person with a particular expression they were inclined to make. As expected, the authors found that happy faces elicited approach and that unpleasant faces (depicting sadness, anger, and disgust) elicited avoidance. Thus, both the behavioral data and the subjective ratings point in the same direction and reveal powerful motivational tendencies in response to social cues. Our experiment using whole-body stepping movements can be considered an ecologically much more valid setup that provides objective measures of tendencies to physically 
approach or avoid another individual displaying a particular facial expression.

Step initiation from a quiet bipedal standing posture is a highly coordinated activity, involving first a rapid lateral weight shift to the stance leg (caused by lifting the swing leg), which is then followed by a leg swing and a wholebody displacement in the desired direction. Forward/ backward stepping from quiet stance has been extensively studied in the motor control literature, and several studies have identified markers in the accompanying center-ofpressure (COP) profile that reveal how the actor organizes this activity in time and place (MacKinnon et al. 2007; Hass et al. 2008). Cued forward/backward stepping involves three phases. The first phase concerns the visual processing of the stimulus. During this phase, the actor is in a more or less quiet standing position and is processing the affective content. During the second phase, the affective content is linked with the instructions, and the actor selects and initiates a forward or backward step. Third, the actor executes the actual step, which involves parameterization of the extent, speed, and force of the step. The question we addressed was whether, and how, the respective phases of voluntary forward or backward steps are influenced by the valence of facial expressions.

With respect to the first phase (affective processing), we expected to find signatures of negative affect in the COP profile preceding the actual step. A number of studies using a quiet stance paradigm have investigated how the emotional and cognitive state of the actor impacts on the ability to maintain balance. COP fluctuations in response to unpleasant visual stimuli typically exhibit reduced excursions and an increase in movement frequency, compared to neutral or pleasant stimuli, which is suggestive of "freezing" (e.g., Azevedo et al. 2005). A recent study (Roelofs et al. 2010a, b) using happy and angry faces, similarly found a reduction in body sway for angry faces, which was accompanied by heart rate deceleration (bradycardia). Thus, we predicted reduced postural excursions with angry faces in the time frame between stimulus presentation and step initiation.

The main hypothesis of this study relates to the second phase, i.e., step selection and initiation. We predicted congruency effects in the step initiation times, that is, faster stepping with approach-to-happy and avoid-to-angry than with the converse assignment. This hypothesis allowed us to test whether the cued stepping paradigm yields the same results with respect to reaction times as numerous previous studies using manual responses. Note also that this setup allowed us to scrutinize an as of yet untested claim in the quiet stance literature regarding affect-primed gait initiation. Some studies have observed shifts in the mean position of the COP (reflecting bodily lean away from the vertical) in an emotion-eliciting context. Those shifts have been interpreted as involuntary signs of gait initiation, in order to approach or avoid an object or situation with positive or negative valence (Carpenter et al. 1999; Maki and McIlroy 1996; Hillman et al. 2004). However, to our knowledge, no study to date has directly tested this hypothesis.

With respect to the third phase of the steps, we expected to find modest effects of affect-congruency on parameters of the actual step execution. Studies examining post-decision motoric processes of arm movements have yielded inconsistent results; for example, Puca et al. (2006) found modest effects of approach-avoidance movements in the force with which the movements were executed. Van Peer et al. (2007) found effects of affect-congruency in both the reaction times and the movement times (MT; the time difference between release of the home button and the pushing of the response button), although the effects were larger for the RTs $\left(\eta^{2}=.37\right)$ than for the MTs $\left(\eta^{2}=.17\right)$. On the other hand, Coombes et al. (2007) only found effects of valence on "central" motor processes but not on "peripheral" motor parameters of arm movements. Finally, Rotteveel and Phaf (2004) observed faster RTs in the affect-congruent than the affect-incongruent condition, but no effects of congruency on the MTs. Given that cued stepping is quite different from manual displacements in terms of force, extent, balance requirements, etc., it seemed worthwhile to study post-decisional characteristics of the steps as well.

In the present experiment, we only tested female participants. Bradley et al. (2001b) found that women displayed stronger defensive reactivity than men, as evidenced by stronger subjective and physiological responses to unpleasant pictures. In addition, Hillman et al. (2004) found that spontaneous postural sway of female participants showed "withdrawal" when viewing unpleasant images (progressive posterior COP displacement), whereas men showed no such effect. Based on these findings, and based on similar considerations of Rotteveel et al. (2004), we decided to test only female subjects, so as to improve the chances of finding the expected effects.

\section{Method}

\section{Participants}

Fifty-two women participated in the study. Ages ranged from 18 to 35 years (mean 22.7 years; $S D 2.5$ years). The study was carried out in full compliance with the principles set out in the Helsinki Declaration and was approved by the local ethics committee prior to its conductance. All participants gave written informed consent. 


\section{Materials}

The stimulus set consisted of eighty full screen images of faces displaying emotive expressions, adopted from the databases of facial expressions of Ekman and Friesen (1976), Martinez and Benavente (1998), Matsumoto and Ekman (1988), and Lundqvist et al. (1998). In total, 40 models (22 men and 18 women) portrayed both a happy and an angry expression. The faces were in black and white and contained no features that were potentially distracting such as facial hair, glasses, hair cuts, and jewelry (e.g., Roelofs et al. 2009a). All the faces displayed direct gaze, i.e., towards the viewer, because a recent study (Roelofs et al. 2010b) found that congruency effects only emerge with direct gaze and not with indirect (averted) gaze.

Participants were positioned on a custom-made strain gauge force plate (dimensions: $1 \mathrm{~m} \times 1 \mathrm{~m}$; sampling frequency: $100 \mathrm{~Hz}$ ), viewing a 17-inch monitor $1 \mathrm{~m}$ placed in front of them on which images were displayed. The force plate consisted of eight force sensors; four measuring forces in the $z$ direction, and two each for the $x$ and $y$ directions. These 8 signals were automatically converted into a COP time series, separate for the anterior-posterior (AP) and the medio-lateral (ML) direction. The sampling frequency of the COP was $100 \mathrm{~Hz}$.

\section{Procedure}

Participants were instructed to categorize the facial expressions, using whole-body single-step movements in a forward or backward direction. In the affect-congruent condition, participants were instructed to make a forward step in response to a happy face and a backward step in response to an angry face. In the incongruent condition, the instructions were reversed (i.e., angry-forward; happybackward). No reference was made in the instructions to congruence and incongruence, or approach and avoidance. Subjects were barefooted. Speed and accuracy were emphasized in the instructions.

Participants were instructed to stand still and adopt a slightly splayed foot stance and to await the arrival of the stimulus. As soon as the visual stimulus appeared, they had to make a fluent step with their right leg followed by their left leg in either the anterior direction (approach) or the posterior direction (avoidance) in response to the valence of the facial expression and to remain stationary until the stimulus disappeared. No emphasis was placed on extent of the steps. During the inter-trial interval, participants had to step back to their starting position and await the next trial.

Each picture was shown for $3 \mathrm{~s}$. After each picture, a black screen was shown for a variable duration of 4-6 s. At the end of the experiment, participants rated the pictures.

The 80 faces were subdivided into four blocks (two for the congruent [C] condition and two for the incongruent [I] condition), which were presented in alternating order. In between each block participants received instructions on the upcoming mapping. Half the subjects performed the blocks in the CICI order, whereas the other half performed the blocks in the ICIC order.

\section{Data analysis}

Figure 1 shows the COP profile of a representative forward (approach; Panel A) and backward (avoidance; Panel B) step.

As can be seen, the COP trace consists of three separate sections, labeled $\mathrm{S} 1$ to $\mathrm{S} 3$. The first section consists of a
Fig. 1 Top view of the COP displacement of a representative participant during a forward step (a) and a backward step (b). $A P$ Anterio-posterior, $M L$ Medio-lateral, $R T$ reaction time. S1 to S3: Sections in the COP trajectory. See text for details
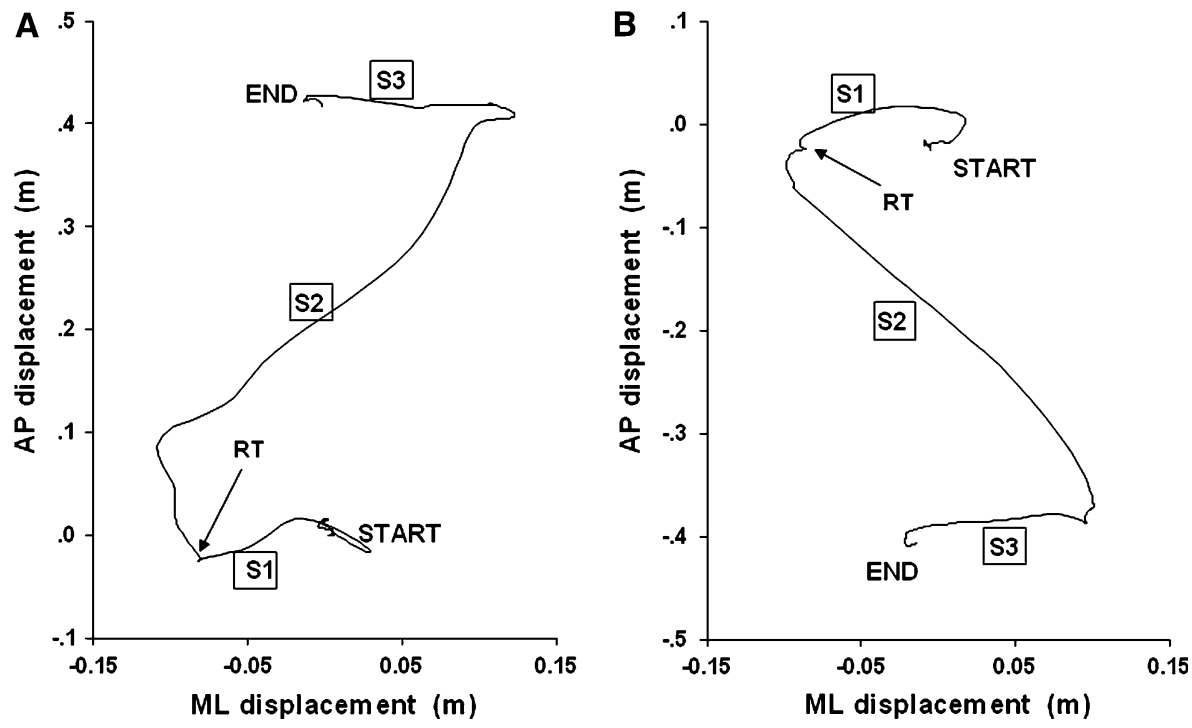
rapid displacement of the COP to the left (stance) limb, caused by changing the distribution of the body weight from underneath both limbs to the left limb. The second section consists of a displacement of the COP in the forward direction (panel A) or backward direction (panel B). This is generated by a swing with the right leg, followed by a landing of the right foot in the new position, immediately followed by lifting the left limb in order to bring it to the new stance position. This sequence of changes explains both the anterio-posterior and medio-lateral changes in the COP. In the third section, the left foot lands next to the right foot, causing a final displacement of the COP in the left direction. During this final phase, the COM undergoes a deceleration, and the body is stabilized in its new position. Note also that forward and backward movements exhibit a qualitatively very similar COP profile, although close scrutiny suggests subtle differences, likely of biomechanical origin.

There are a number of relevant spatio-temporal parameters from the COP profile associated with the steps.

\section{Postural immobility}

This measure refers to initial postural reactions prior to onset of the step. Unpleasant visual stimuli can induce reductions in spontaneous postural sway, which is reminiscent of "freezing". Postural immobility was quantified as the combined length of the COP trace (sway path length) in the 0 to $250-\mathrm{ms}$ interval following onset of the stimulus. This range was chosen as it represents visual processing of the faces, prior to actual initiation of the step.

\section{Reaction time}

RT is classically defined as the time interval between stimulus onset and the first overt change in behavior. In our study, participants always initiated their step with the right limb, so in principle, they could have initiated the step at stimulus onset by lifting their right limb, prior to (or simultaneous with) selection of the subsequent step direction. From the COP traces, it can be seen that each step involves a clear medio-lateral displacement of the COP, which is then followed by a movement of the COP in the anterior or posterior direction. We reasoned that a directional change in the COP coincides with a clear drop in the velocity profile of the COP. Figure 2 represents the velocity profiles of the same trials as shown in Fig. 1. Note that the COP profiles exhibit three distinct peaks, corresponding to the three sections shown in Fig. 1. The change in movement direction corresponds to the valley between the first and second peak, so we defined RT as the moment at which the velocity between these peaks reaches its minimum.
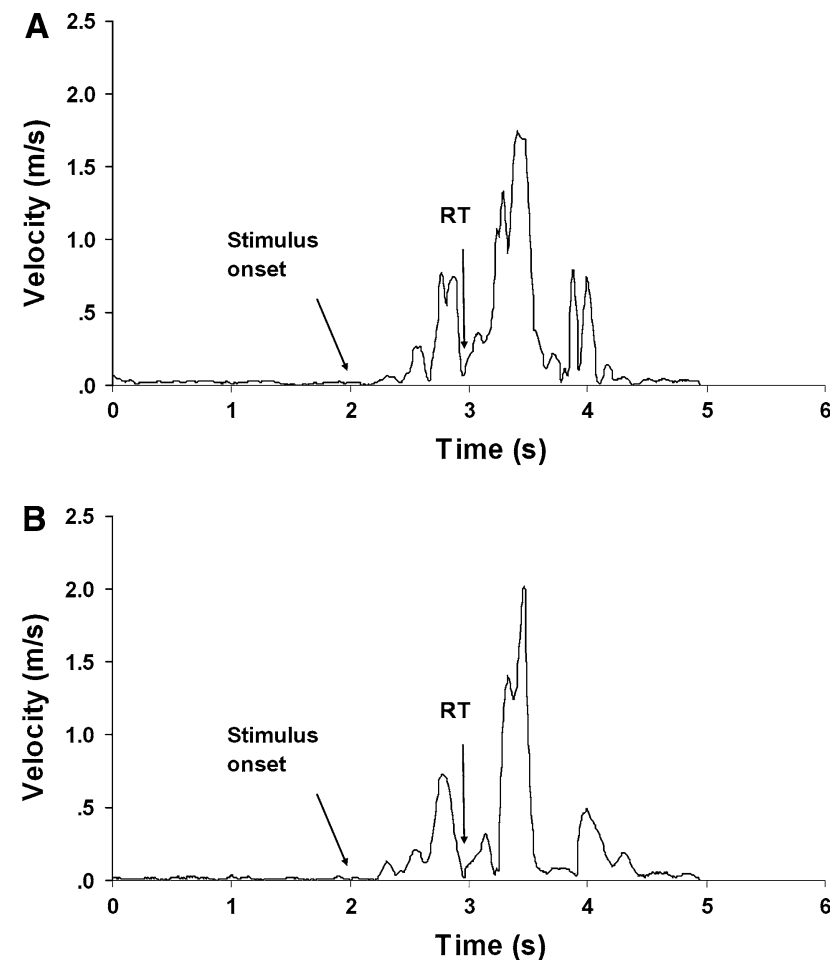

Fig. 2 Velocity profiles accompanying the steps shown in Fig. 1. RT reaction time

Step execution: movement time

Movement time (MT) is the time interval between the reaction time and the moment at which the COP reached its final position.

Step execution: peak velocity

Peak velocity is the maximum speed $(\mathrm{m} / \mathrm{s})$ obtained during the steps and is related to fast acceleration in the forward or backward direction (see section S2 of Fig. 1).

Statistical analysis

Incorrect trials were first identified and deleted. Trials were counted as errors when (a) a step was made in the wrong direction, (b) a step was made with the left limb, (c) the RT fell outside the range 250-2,000 ms, (d) participants failed to adopt a quiet standing posture prior to the stimulus, defined as COP velocity $>10 \mathrm{~cm} / \mathrm{s}$, or (e) participants did not come to a complete standstill following step execution. In addition, the first three trials within each of the four blocks were not analyzed, to prevent potential carry-over effects from the previous block.

The COP parameters were analyzed using separate 2 (movement direction; forward vs. backward) $\times 2$ (emotion; happy vs. angry facial expression) analyses of 
variance (ANOVA). Significant effects were explored further using paired-sampled $t$ tests. We adopted a $P$-value of .05 for all analyses. Effect sizes $(E S)$ of main and interaction effects were expressed as partial eta squared $\left(\eta^{2}\right)$.

Note that effects of mapping will show up as an interaction of facial expression and movement direction, meaning that the pleasant-forward/angry-backward (congruent) combination is expected to yield different performance than the pleasant-backward/angry-forward (incongruent) combination.

\section{Results}

One participant exhibited an excessive number of errors ( $>20 \%$; mostly due to excessive pre-stepping body sway) and her data were excluded from further analyses. Of the remaining trials 169 (4.9\%) were qualified as errors, using the criteria above. Again, most errors were due to excessive body sway prior to the stimulus. In total, only 18 errors $(0.5 \%)$ involved a step made in the wrong direction. The distribution of errors was as follows: congruent-happy: 3 ; congruent-angry: 3 ; incongruent-happy: 6 ; incongruentangry: 6. Although these numbers reveal a tendency to execute an affect-congruent step, these numbers were too small to perform a reliable test of statistical significance.

\section{Postural immobility}

The ANOVA for early postural immobility (sway path length) revealed a main effect of movement direction, $F(1,50)=8.57, P<.01, \eta^{2}=.15$, which was superseded by a significant movement direction by emotion interaction, $F(1,50)=8.85, P<.01, \eta^{2}=.15$. Exploration of the interaction revealed that with forward steps, the length of the sway path was reduced with angry faces compared to happy faces, $t(50)=2.80, P<.01$, (5.3 vs. $6.4 \mathrm{~mm}$ ), whereas with backward steps, sway path length was reduced with happy faces compared to angry faces, $t(50)=2.04, P<.05$ (5.1 vs. $5.6 \mathrm{~mm}$ ). In other words, in the incongruent conditions, postural sway was smaller in magnitude directly after stimulus presentation than in the congruent conditions, regardless of the valence of the facial expression.

\section{Reaction time}

The main effect of emotion was significant, $F(1$, $50)=8.60, P<.01, \eta^{2}=.15$, as was the main effect of movement direction, $F(1,50)=36.34, P<.001$, $\eta^{2}=.42$. These main effects were superseded by a significant movement direction by emotion interaction, $F(1$,

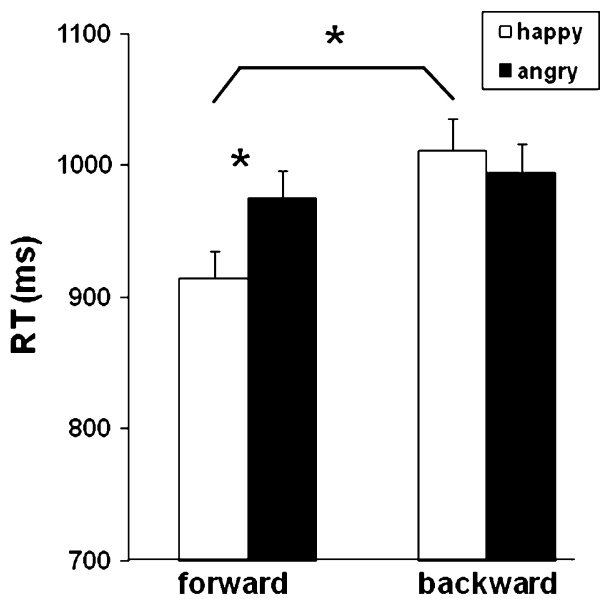

Fig. 3 Mean reaction times (RTs) for happy and angry steps, as a function of step direction (forward/angry). $* P<.05$. Error bars denote standard errors of the mean

50) $=13.63, \quad P<.001, \quad \eta^{2}=.21 . \quad$ Follow-up analysis revealed that forward steps were initiated faster in response to happy faces than in response to angry faces, $t(50)=4.71, P<.001$, whereas this difference was not significant for backward steps. In addition, we found that forward steps were initiated faster than backward steps in response to happy faces, $t(50)=6.69, P<.001$, whereas this difference was not significant for angry faces $(P>.1)$. The means are shown in Fig. 3.

\section{Movement time}

The ANOVA only revealed a main effect of movement direction, $F(1,50)=39.98, P<.01, \eta^{2}=.44$, which was caused by faster backward than forward stepping (760 vs. $1,000 \mathrm{~ms}$, respectively).

\section{Peak velocity}

All effects were significant; there were main effects of movement direction, $F(1,50)=13.96, P<.001$, $\eta^{2}=.22, \quad$ and emotion, $F(1,50)=6.85, \quad P<.01$, $\eta^{2}=.12$, and a significant movement direction by emotion interaction, $F(1,50)=12.25, P<.001, \eta^{2}=.20$. Followup analysis revealed that the difference between happy and angry faces was significant for the backward steps, $t(50)=4.12, P<.001$. The means $(+$ standard errors of the mean) were $1.65(.054)$ versus $1.58(.054) \mathrm{m} / \mathrm{s}$, respectively. The same contrast for the forward steps, however, was not significant; $1.50(.048)$ versus $1.52(.046)$ $\mathrm{m} / \mathrm{s}$, respectively. Furthermore, the difference between forward and backward steps was significant for happy faces, $t(50)=4.44, P<.001(1.50$ vs. $1.65 \mathrm{~m} / \mathrm{s})$, and also for angry faces, $t(50)=2.26, P<.05(1.52$ vs. $1.58 \mathrm{~m} / \mathrm{s})$, yet smaller in magnitude. 


\section{Discussion}

In the present study, we adopted a novel version of the approach-avoidance task, involving whole-body stepping movements toward or away from facial expressions. This setup was chosen first because of its greater ecological validity in the context of social cues, at least compared to unimanual responses (Koch et al. 2009). Second, the setup combines posturographic recordings with a choice RT paradigm, thereby bringing together the study of affectcongruency effects with that of the influence of emotion on the regulation of balance.

We hypothesized that angry or happy facial expressions, combined with the instruction to move in a particular direction, affect all stages in the organization of a step. This was indeed found to be the case.

First, we found evidence of reduced postural mobility in the first $250 \mathrm{~ms}$ after onset of the stimulus. This reduction in sway was not linked with the facial expression, as we had expected, but with the S-R assignment. Participants displayed greater immobility during the incongruent blocks of trials than during the congruent blocks, regardless of the valence of the facial expressions. We believe this was due to a greater demand for attentional resources needed to store, retrieve, and implement the incongruent instructions, relative to the congruent instructions. Researchers studying changes in postural control have pointed out that postural immobility may not be (exclusively) due to valence, but (also) to the attentional demands of the task; for example, Dault et al. (2001) found that various working memory (WM) tasks, such as word categorization and random number generation, performed concurrently with quiet standing, all led to a reduction in postural sway. It was hypothesized that adopting a quiet and stiff posture (using co-contracting of antagonistic lower leg muscle pairs) is less attention demanding and may thereby free attentional resources to perform the attention demanding secondary task. A similar conclusion was reached by Stins et al. (2011), who directly compared the effects of cognitive and affective perturbations on postural sway. Thus, some of the literature on posture-cognition dual-tasking indicates that attention demanding cognitive tasks can induce a reduction in body sway. It is doubtful whether such a reduction in sway constitutes genuine "freeze", as freezing behavior is classically defined as a threat-induced cessation of bodily motion associated with bradycardia, yet with a clear attentional component (Roelofs et al. 2010a). It is interesting to note that attention has also been found to play a role in step initiation. Melzer and Oddsson (2004) employed a paradigm whereby healthy young and elderly individuals were instructed to initiate a step upon detection of a cutaneous cue (a tap to the heel). Importantly, in some conditions, subjects stood while they performed an attention-demanding Stroop task. It was found that various parameters of the COP and the ground reaction forces of the step were affected by this attentional manipulation, although it should be noted that Melzer and Oddsson (2004) partitioned the COP profile in a manner different from ours. This finding is in line with our interpretation that affect-incongruency places an attentional burden on the balance system, which becomes manifest in various phases of the step.

Second, we partially replicated previous AA effects obtained in the manual choice RT paradigm. The movement direction by emotion interaction revealed that the affect-congruent S-R assignment yielded faster step initiation times than the affect-incongruent assignment, but only in certain conditions. Inspection of the cell means revealed that participants needed more time to initiate a forward step towards an angry face than towards a smiling face. With backward steps, RTs were somewhat faster with angry faces than with smiling faces, although this difference failed to reach significance. Furthermore, the results suggest clear congruency effects with smiling faces, but not with angry faces, thereby replicating the findings of Seidel et al. (2010). This set of results suggests that social cues trigger automatic response tendencies, because-in the affectincongruent condition-subjects needed more time to inhibit these tendencies and select a counter-intuitive response. This is an important finding, as this is, to our knowledge, the first demonstration that social cues prime whole-body displacements. Our data revealed an asymmetry between reactions to smiling faces and angry faces, similar to Seidel et al. (2010). In contrast, other studies (e.g., Rotteveel and Phaf 2004) found clear symmetric effects in the RTs, namely equally-sized congruency effects with happy and angry faces. The reason for this discrepancy is not clear, but asymmetric results in the AA task have been similarly reported (e.g., Roelofs et al., 2009a), and they could be related to the fact that affective reactions to expressions of anger and happiness are not simply mirror opposites.

Third, we found that the execution of the steps was mainly affected by step direction, with large differences in spatio-temporal parameters between forward and backward steps. Of course, forward and backward steps are clearly different in terms of the patterns of muscular activation accompanying the movements and in terms of the availability of visual information to guide the movements. We found only modest effects of emotional expression for the peak velocity of the step, suggesting that happy faces triggered greater velocity changes of the step than angry faces. This could be due to a more conservative or cautious movement strategy with angry faces, but this issue requires further testing. However, the results seem to confirm the intuition of Coombes et al. (2007) that valenced stimuli have hardly any influence on motor execution. 
Note that in our experiment, we implicitly adopted a basic motivational assumption, namely, that people are motivated to approach pleasant objects or individuals and to avoid unpleasant ones. This so-called hedonic principle (e.g., Higgins 1997) is at the basis of many psychological theories, but may be a gross over-simplification of actual human motivated behavior. As pointed out by Higgins (1997), approach/avoidance behavior can only be understood within the context of the regulatory focus adopted by each individual. Simply put, an individual may strive to maximize gain (promotion focus) or to minimize loss (prevention focus). A promotion focus or prevention focus can either be related to a stable personality trait or can be induced experimentally. With respect to approach/avoidance tasks, it has been found that approach or avoidance behavior (operationalized as the strength of static arm flexion or extension) was dependent on regulatory focus (Förster et al. 1998, 2001). In our present study, we did not manipulate regulatory focus, but it could well be that individuals who happen to be fearful of angry faces perform the task using a prevention focus, and that individuals who happen to be particularly attracted to smiling faces perform the task using a promotion focus. Our research paradigm opens up the possibility of examining how regulatory focus constrains the spatio-temporal organization of goal-directed behavior.

To conclude, we found several effects of facial expression in posturographic characteristics of stepping movements. The current set of results extends previous studies that examined manual responses to facial expressions (e.g., Roelofs et al. 2009b, 2010b). These latter studies observed not only affect-congruency effects but also that the strength of the effect was modulated by subject variables such as social anxiety disorder. We used a highly ecologically valid setup, involving physical approach and avoidance of potent social cues. Stepping forward entails bringing the body into the vicinity of someone else. When approaching a friendly face, this may invite physical contact such as a hug or a hand shake, and it may facilitate communication. But when approaching an angry face, one exposes oneself to potential physical and verbal abuse. Stepping backward, on the other hand, gives rise to the opposite behavioral possibilities. Thus, a step in a particular direction creates the opportunity for a whole range of (wanted or unwanted) social encounters and can thus be considered of utmost importance for social interaction. We found that various phases in the spatio-temporal organization of the steps were related to social cues, thereby providing a crucial empirical link between theories of socially induced action tendencies and the organization of the actual motoric output. Future studies should adopt a wider range of emotional expressions (cf. Seidel et al. 2010) and also include a set of neutral stimuli (cf. Roelofs et al. 2010a) that can be used as a baseline against which to compare approach and avoidance tendencies and their relative impact on behavior.

Open Access This article is distributed under the terms of the Creative Commons Attribution Noncommercial License which permits any noncommercial use, distribution, and reproduction in any medium, provided the original author(s) and source are credited.

\section{References}

Azevedo TM, Volchan E, Imbiriba LA, Rodrigues EC, Oliveira JM, Oliveira LF, Lutterbach LG, Vargas CD (2005) A freezinglike posture to pictures of mutilation. Psychophysiology 42: 255-260

Bradley MM, Codispoti M, Cuthbert BN, Lang PJ (2001a) Emotion and motivation I: defensive and appetitive reactions in picture processing. Emotion 1:276-298

Bradley MM, Codispoti M, Sabatinelli D, Lang PJ (2001b) Emotion and motivation II: sex differences in picture processing. Emotion $1: 300-319$

Carpenter MG, Frank JS, Silcher CP (1999) Surface height effects on postural control: a hypothesis for a stiffness strategy for stance. J Vestib Res 9:277-286

Chen M, Bargh JA (1999) Consequences of automatic evaluation: immediate behavioral predispositions to approach or avoid the stimulus. Pers Soc Psychol Bull 25:215-224

Coombes SA, Cauraugh JH, Janelle CM (2007) Dissociating motivational direction and affective valence. Psychol Sci 18:938-942

Dault MC, Frank JS, Allard F (2001) Influence of a visuo-spatial, verbal and central executive working memory task on postural control. Gait Posture 14:110-116

Eder AB, Rothermund K (2008) When do motor behaviors (mis)match affective stimuli? An evaluative coding view of approach and avoidance reactions. J Exp Psychol General 137:262-281

Ekman P, Friesen WV (1976) Pictures of facial affect. Consulting Psychologist Press, Palo Alto

Förster J, Higgins ET, Idson LC (1998) Approach and avoidance strength during goal attainment: regulatory focus and the "goal looms larger" effect. J Pers Soc Psychol 75:1115-1131

Förster J, Grant H, Idson CI, Higgins ET (2001) Success/failure feedback, expectancies, and approach/avoidance motivation: how regulatory focus moderates classic relations. J Exp Soc Psychol 37:253-260

Hass CJ, Waddell DE, Wolf SL, Juncos JL, Gregor RJ (2008) Gait initiation in older adults with postural instability. Clin Biomech 23:743-753

Higgins ET (1997) Beyond pleasure and pain. Am Psychol 52:12801300

Hillman CH, Rosengren KS, Smith DP (2004) Emotion and motivated behavior: postural adjustments to affective picture viewing. Biol Psychol 66:51-62

Koch S, Holland RW, Hengstler M, van Knippenberg A (2009) Body locomotion as regulatory process. Psychol Sci 20:549-550

Krieglmeyer R, Deutsch R, de Houwer J, de Raedt R (2010) Being moved: valence activates approach-avoidance behavior independently of evaluation and approach-avoidance intentions. Psychol Sci 21:607-613

Lang PJ, Bradley MM, Cuthbert BN (1998) Emotion, motivation, and anxiety: brain mechanisms and psychophysiology. Biol Psychiatry $44: 1248-1263$

Lavender T, Hommel B (2007) Affect and action: towards an eventcoding account. Cogn Emotion 21:1270-1296 
Lundqvist D, Flykt A, Öhman A (1998) The Karolinska directed emotional faces-KDEF, CD ROM from Department of Clinical Neuroscience, Psychology section, Karolinska Institute, Sweden

MacKinnon CD, Bissig D, Chiusano J, Miller E, Rudnick L, Jager C, Zhang Y, Mille M-L, Rogers MW (2007) Preparation of anticipatory postural adjustments prior to stepping. J Neurophysiol 97:4368-4379

Maki BE, McIlroy WE (1996) Influence of arousal and attention on the control of postural sway. J Vestib Res 6:53-59

Markman AB, Brendl CM (2005) Constraining theories of embodied cognition. Psychol Sci 16:6-10

Marsh AA, Ambady N, Kleck RE (2005) The effects of fear and anger facial expressions on approach and avoidance-related behaviors. Emotion 5:119-124

Martinez AM, Benavente R (1998) The AR face database (CVC tech. Rep. No. 24)

Matsumoto D, Ekman P (1988) Japanese and Caucasian facial expressions of emotion (JACFEE) [Slides]. University of California, Human Interaction Laboratory, San Francisco

Melzer I, Oddsson LIE (2004) The effect of a cognitive task on voluntary step execution in healthy elderly and young individuals. J Am Geriatr Soc 52:1255-1262

Puca RM, Rinkenauer G, Breidenstein C (2006) Individual differences in approach and avoidance movements: how the avoidance motive influences response force. J Pers 74:979-1014

Rinck M, Becker ES (2007) Approach and avoidance in fear of spiders. J Behav Ther Exp Psychiatry 38:105-120

Roelofs K, Elzinga BM, Rotteveel M (2005) The effects of stressinduced cortisol responses on approach-avoidance behavior. Psychoneuroendocrinology 30:665-677
Roelofs K, Minelli A, Mars RB, van Peer J, Toni I (2009a) On the neural control of social motivational behaviour. Soc Cogn Affect Neurosci 4:50-58

Roelofs K, van Peer J, Berretty E, de Jong P, Spinhoven P, Elzinga BM (2009b) Hypothalamus-pituitary-adrenal axis hyperresponsiveness is associated with increased social avoidance behavior in social phobia. Biol Psychiatry 65:336-343

Roelofs K, Hagenaars M, Stins JF (2010a) Facing freeze: social threat induces bodily freeze in humans. Psychol Sci 21:1575-1581

Roelofs K, Putnam P, Schouten S, Lange W-G, Volman I, Rinck M (2010b) Gaze direction differentially affects avoidance tendencies to happy and angry faces in socially anxious individuals. Behav Res Ther 48:290-294

Rotteveel M, Phaf RH (2004) Automatic affective evaluation does not automatically predispose for arm flexion and extension. Emotion 4:156-172

Seidel E-M, Habel U, Kirschner M, Gur RC, Derntl B (2010) The impact of facial emotional expressions on behavioral tendencies in women and men. J Exp Psychol Hum Percept Perform 36:500-507

Stins JF, Roerdink M, Beek PJ (2011) To freeze or not to freeze: Affective and cognitive perturbations have markedly different effects on postural control. Hum Mov Sci 30:190-202

van Peer JM, Roelofs K, Rotteveel M, van Dijk JG, Spinhoven Ph, Ridderinkhof KR (2007) The effects of cortisol administration on approach-avoidance behavior: an event-related potential study. Biol Psychol 76:135-146

Van Peer JM, Rotteveel M, Spinhoven Ph, Tollenaar MS, Roelofs K (2010) Affect-congruent approach and withdrawal movements of happy and angry faces facilitate affective categorisation. Cogn Emotion 24:863-875 